\title{
ANAESTHETIC CONCERNS IN A CASE OF APERT SYNDROME POSTED FOR SYNDACTYLY RELEASE
}

\author{
Savitri Durgaprasad Kabade, Karthik Shivani Lokeshappa², Venkatesh Yshya ${ }^{3}$, Elizabeth Wilson ${ }^{4}$
}

${ }^{1}$ Associate Professor, Department of Anaesthesiology, Karnataka Institute of Medical Sciences, Hubli.

2 Post Graduate, Department of Anaesthesiology, Karnataka Institute of Medical Sciences, Hubli.

${ }^{3}$ Post Graduate, Department of Anaesthesiology, Karnataka Institute of Medical Sciences, Hubli.

${ }^{4}$ Post Graduate, Department of Anaesthesiology, Karnataka Institute of Medical Sciences, Hubli.

\begin{abstract}
Apert syndrome is an autosomal dominant disease presenting with craniosynostosis, midface hypoplasia and syndactyly. Children with Apert syndrome usually present to the operating room for craniofacial and extremity operations. During anaesthe sia, difficult intubation and ventilation is expected because of abnormal airways. In case of Apert syndrome, anaesthetists must be prepared for intubation difficulties, airway, ventilation problems and even visceral anomalies. We report a case of Apert syndrome referred to us for endoscopy of upper respiratory tract and syndactyly release. This article will focus on the difficulties and complications related to the anaesthetic management.
\end{abstract}

\section{KEYWORDS}

Apert Syndrome, Syndactyly, Difficult Airway.

HOW TO CITE THIS ARTICLE: Kabade SD, Lokeshappa KS, Yshya V, et al. Anaesthetic concerns in a case of apert syndrome posted for syndactyly release. J. Evolution Med. Dent. Sci. 2016;5(59):4145-4147, DOI: 10.14260/jemds/2016/947

\section{INTRODUCTION}

Apert syndrome is a rare congenital autosomal dominant disease with an incidence of 1 per 160,000 live births. The syndrome is characterized by brachycephaly, craniosynostosis, midface hypoplasia, hypertelorism, choanal stenosis, multidigit hand and foot syndactyly. Patients present to the operating room for craniofacial and extremity operations. ${ }^{1}$ Children with Apert syndrome have a much higher incidence of perioperative airway and respiratory complications than other children.1,2 We report our experience in the anaesthetic management of a case of Apert syndrome referred to us for endoscopy of upper respiratory tract and syndactyly release procedures.

\section{CASE REPORT}

A 15-month-old female baby, born by normal vaginal delivery at full term, was posted for left hand and foot syndactyly release. The child was breathing through mouth since birth, hence the child was referred to Dept. of Ear, Nose and Throat for evaluation. Baby was unable to crawl and could not understand simple words, suggestive of delayed milestones for her age. She had repeated episodes of respiratory tract infection, which was treated with antibiotics. Family history was not significant. On physical examination her vitals were recorded as: pulse-100/min, NIBP-96/64 mmHg, RR-24/min. Cardiorespiratory systems examination were normal. She was having mid-facial hypoplasia involving maxillary and zygomatic bones with orbital proptosis, hypertelorism (Figure 1), brachycephaly, high-arched palate, cleft palate (Figure 2) and all limbs showed fusion of multiple digits (Figure 3 and 4).

Financial or Other, Competing Interest: None.

Submission 13-06-2016, Peer Review 09-07-2016,

Acceptance 15-07-2016, Published 25-07-2016.

Corresponding Author:

Dr. Savitri Durgaprasad Kabade,

Associate Professor,

Department of Anaesthesiology,

Karnataka Institute of Medical Sciences,

Hubli-580022.

E-mail: sdkabade20@gmail.com

DOI: $10.14260 /$ jemds/2016/947
She was diagnosed as a case of Apert syndrome. Her preoperative investigations were: $\mathrm{Hb}-10.5 \mathrm{~g} / \mathrm{dL}$, platelet count - 4 lakhs $/ \mathrm{mm}^{3}$ with normal renal and liver function tests. Chest $\mathrm{X}$-ray, 2D Echocardiogram and Ultrasound of abdomen were normal. Consent was taken from parents and baby was advised to be NPO for 4 hours before endoscopy.

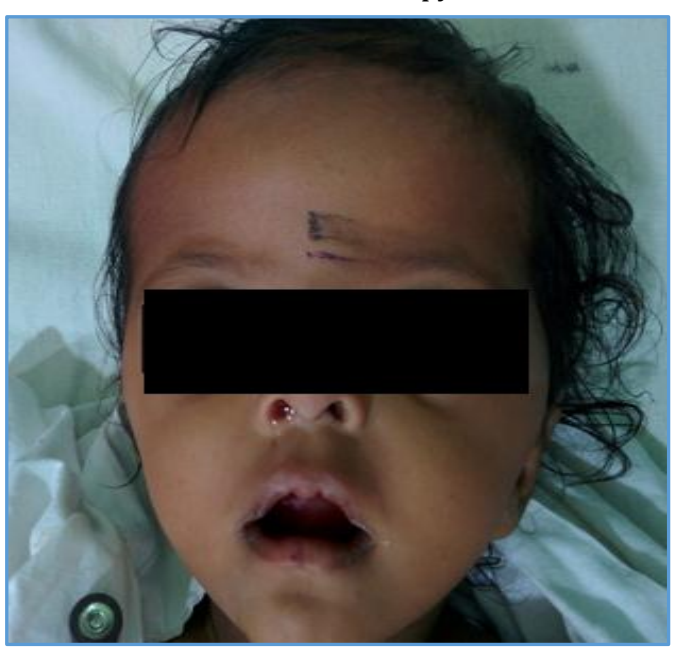

Fig. 1: Hypertelorism Figure

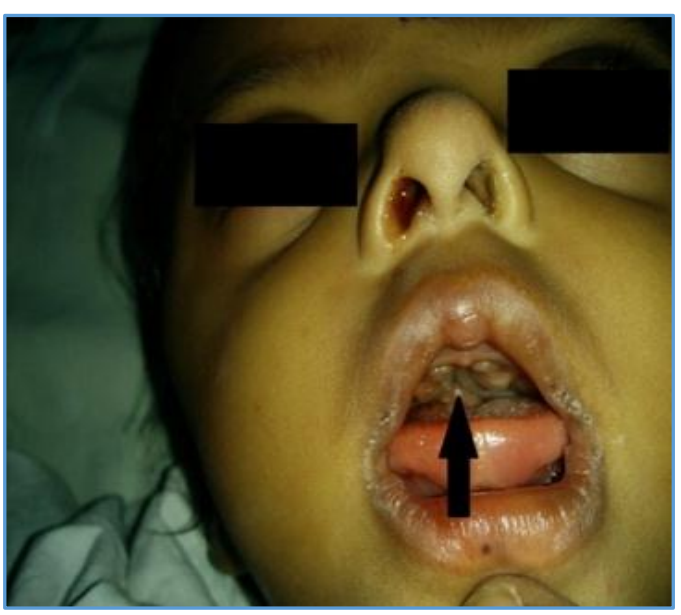

Fig. 2: Cleft Palate (Arrow) 


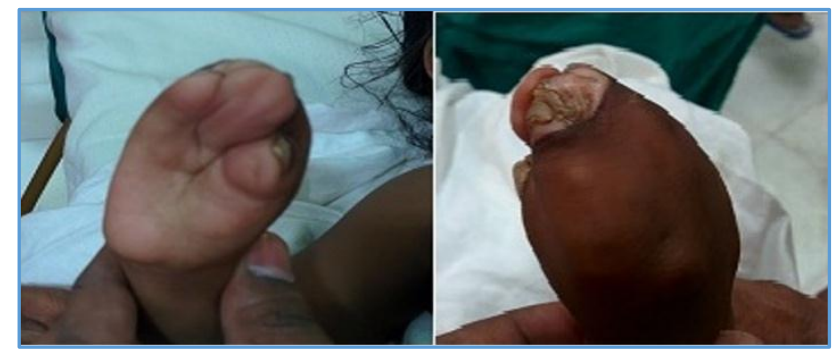

Fig. 3: Syndactyly of Upper Limb (Rose Bud Type)

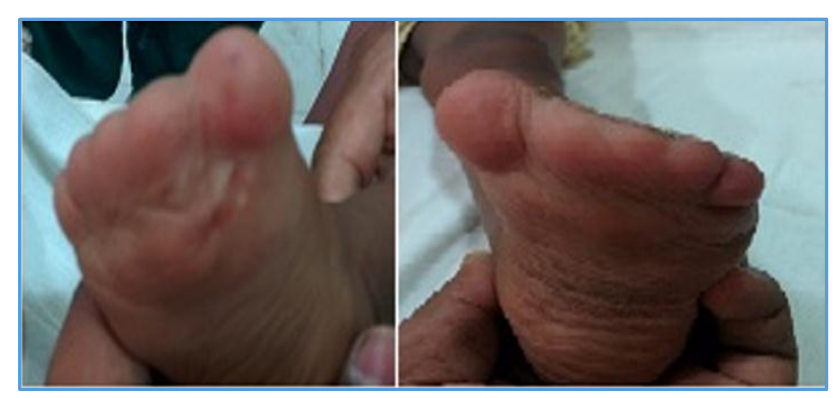

Fig. 4: Syndactyly of Lower Limb

On the day of endoscopy, child was pre-medicated with Inj. Glycopyrrolate $(0.005 \mathrm{mg} / \mathrm{kg})$. Baseline vitals were recorded: PR-110/min, NIBP-100/68 mmHg, RR-20/min, Spo2-98\% at room air; 24-G IV cannula was secured on right upper limb. Child was sedated with Inj. Midazolam $(0.05 \mathrm{mg} / \mathrm{kg})$ and Inj. ketamine $(2 \mathrm{mg} / \mathrm{kg})$. Anaesthesia was maintained with 02 , N2O with patient on spontaneous respiration. Vitals were stable throughout the procedure. Duration of procedure was 15 minutes. In post-anaesthetic period, child was awake with stable vitals. There was no significant airway anomalies except mild tracheal stenosis.

Three days later she was posted for release of syndactyly in left hand and foot. Consent was taken from parents and advised NPO for 4 hours before surgery. Premedication was given with oral midazolam syrup $(0.5 \mathrm{mg} / \mathrm{kg}) 30$ minutes before the procedure. After achieving a score of 4 on Ramsay sedation score, she was easily separated from her parents and shifted to operating table. General anaesthesia was planned for the procedure. Difficult airway cart was kept ready including laryngeal masks and emergency tracheostomy sets. In the operating room standard monitors were attached. Intravenous line was secured with 24-G cannula on right upper limb and Isolyte-P infusion started as per Holliday-Segar's formula. Anaesthesia was induced with glycopyrrolate $(0.005 \mathrm{mg} / \mathrm{kg})$, fentanyl $(2 \mu \mathrm{g} / \mathrm{kg})$ and thiopentone sodium $(5 \mathrm{mg} / \mathrm{kg})$ and after ensuring smooth mask ventilation, atracurium $7.5 \mathrm{mg}$ was given. Child was intubated with $3.0 \mathrm{~mm}$ ID uncuffed endotracheal tube. Intraoperative anaesthesia was maintained with $\mathrm{N}_{2} \mathrm{O}, \mathrm{O}_{2}$ and isoflurane. Blood pressure, pulse, SpO2, ECG, $\mathrm{EtCO}_{2}$ and temperature monitoring were done throughout the procedure and were stable. Surgery lasted for 2 hours and intraoperative course was uneventful. At the end of surgery, child was extubated after oral suctioning. Post-operative analgesia was provided with per rectal paracetamol suppository. Baby was shifted to paediatric intensive care unit for observation where she had an uneventful stay.

\section{DISCUSSION}

Apert syndrome is one of the syndrome of craniosynostosis. It is defined by the triad of craniosynostosis, midface hypoplasia and syndactyly of the hands and feet. Associated features may include developmental delay, proptosis, hypertelorism, cleft palate, dental malocclusion, cervical spine fusion and cardiorespiratory, renal and gastrointestinal anomalies. ${ }^{3}$

Genetically, the defect is in chromosome 10 affecting fibroblast growth factor receptor 2 gene. This mainly affects digits and cranium causing fusion of multiple digits of both upper and lower limbs and premature fusion of cranial sutures. ${ }^{4}$

Syndactyly or Webbing of the digits is caused by failure, during the sixth to eighth weeks of intrauterine life of the usual longitudinal interdigital necrosis that normally separates the fingers. 5

There is no definite recommendation for general or regional anaesthesia and also there are no known contraindications to specific anaesthetic agent and drug. ${ }^{1}$

These children require a number of different operative procedures and anaesthetists face multiple problems while dealing with them, first of it being the airway management. Airway dysmorphism carries a risk of difficult mask ventilation and intubation due to mid-face hypoplasia. ${ }^{6}$ Cartilaginous abnormalities of the trachea, fusion of the cervical vertebra, tracheal stenosis and angular deviation of the trachea may contribute to difficult intubation in these patients. Inside the mouth the challenge to airway instrumentation is increased by tooth crowding, a narrow $\mathrm{V}$-shaped maxillary alveolar arch and a cleft or high arched palate.1,7 In our case, child was having cleft palate and mild tracheal stenosis. So we could pass an endotracheal tube with a size of $3.0 \mathrm{~mm}$ with due precautions for cleft palate.

Craniofacial anomalies are often associated with obstructive sleep apnoea and therefore are prone obstruction on induction and emergence. ${ }^{8}$ The incidence of respiratory complications is $33 \%$ in these patients. Patients with Apert Syndrome may have lower airway compromise due to stiff or vertically fused tracheal rings. During coughing, the resulting inability to narrow the trachea to create high velocity airflow impairs clearance of secretions. The accumulation of such secretions during anaesthesia could make a monophonic 'wheezing' sound during anaesthesia. ${ }^{9}$

Abnormal skull development may lead to shallow orbits causing protrusion of the eyes and inability to close eyes, known as exorbitism. Due to inadequate lid closure eyes are particularly susceptible to damage and hence eyes should be lubricated, taped and padded. Consideration should be given to avoid pressure points. ${ }^{1}$ In our case as she was having proptosis, eyes were lubricated with tear drops and padded.

Another challenge for the anaesthetist is intravenous access. Limb deformity and multiple operative procedures make intravenous access more difficult. As a result some anaesthetists feel that for short procedures, e.g. change of dressings or CT, intravenous access is not mandatory. In an emergency, an intraosseous or intramuscular route can be used. $^{2}$

These patients tend to sweat a lot and unlike other patients undergoing syndactyly surgery do not appear to need warming when undergoing peripheral surgery to their limbs. If they are actively warmed there is a risk of pyrexia, so temperature should always be monitored. ${ }^{1}$ 
Regional anaesthesia is preferred over general anaesthesia due to airway and respiratory problems. In addition, regional anaesthesia reduces perioperative opioid requirements and thus reduces the opioid side effects like deep sedation, respiratory depression and nausea and vomiting. Regional anaesthesia could be difficult in these patients due to anatomical variations in shoulder joint and related structures and ultrasound guidance may help in these patients. ${ }^{1}$ In our case as surgery was planned for both upper and lower limb, we opted for general anaesthesia.

\section{CONCLUSION}

Anaesthetic management of a patient with Apert syndrome may be challenging, because of upper airway anomalies. So thorough preoperative evaluation, a proper anaesthesia plan as well as a backup for difficult airway management is very important in the management of children with Apert's syndrome.

\section{REFERENCES}

1. Bansal T, Jaiswal R, Hooda $\mathrm{S}$, et al. Apert syndrome: anaesthetic concerns and challenges. Egyptian Journal of Anaesthesia 2015;31(1):85-7.

2. Barnett S, Moloney C, Bingham R. Perioperative complications in children with apert's syndrome: a review of 509 anaesthetics. Paediatr Anesth 2011;21(1):72-7.
3. Palmisano BW. Anesthesia for plastic surgery. In: Gregory GA, ed. Pediatric Anesthesia. $3^{\text {rd }}$ edn. New York: Churchill Livingstone 1994:699-740.

4. Chen L, Li D, Li C, et al. A ser250trp (corrected) substitution in mouse fibroblast growth factor receptor 2 (Fgfr2) results in craniosynostosis. Bone 2003;33(20): 169-78.

5. Upton J. Apert syndrome. Classification and pathologic anatomy of limb anomalies. Clinics in Plastic Surgery 1991;18(2):321-55.

6. Pardos PC, Pazos JC. Management of the airway in an infant with apert syndrome. Rev Esp Anestesiol Reanim 2000;47(2):97-8.

7. Morris GP, Cooper MG. Difficult tracheal intubation following midface distraction surgery. Pediatr Anesth 2000;10(1):99-102.

8. Perkins JA, Sie KCY, Milczuk H, et al. Airway management in children with craniofacial anomalies. Cleft Palate Craniofac J 1997;34(2):135-40.

9. Elwood T, Sarathy PV, Geiduschek JM, et al. Respiratory complications during anaesthesia in apert syndrome. Pediatr Anesth 2001;11(6):701-3. 